Supplementary Information of

\title{
Improving predictability of high ozone episodes through dynamic boundary conditions, emission refresh and chemical data assimilation during the Long Island Sound Tropospheric Ozone Study (LISTOS) field campaign
}

Siqi Ma ${ }^{1,2}$, Daniel Tong ${ }^{1,3^{*}}$, Lok Lamsal ${ }^{4,5}$, Julian Wang $^{6 *}$, Xuelei Zhang ${ }^{3}$, Youhua Tang ${ }^{3,6}$, Rick Saylor ${ }^{6}$, Tianfeng Chai ${ }^{4}$, Pius Lee ${ }^{6}$, Patrick Campbell ${ }^{3,6}$, Barry Baker ${ }^{3,6}$, Shobha Kondragunta ${ }^{7}$, Laura Judd ${ }^{8}$, Timothy A. Berkoff $^{8}$, Scott J. Janz ${ }^{4}$, and Ivanka Stajner ${ }^{9}$

${ }^{1}$ Department of Atmospheric, Oceanic and Earth Sciences, George Mason University, Fairfax, VA 22030 USA

${ }^{2}$ National Research Council, hosted by the National Oceanic and Atmospheric Administration Air Resources Lab, College Park, MD 20740 USA

${ }^{3}$ Center for Spatial Information Science and Systems, George Mason University, Fairfax, VA 22030 USA

${ }^{4}$ Atmospheric Chemistry and Dynamics Laboratory, NASA Goddard Space Flight Center, MD 20771 USA

${ }^{5}$ Universities Space Research Association, Columbia, MD 21046 USA

${ }^{6}$ National Oceanic and Atmospheric Administration (NOAA) Air Resources Laboratory, College Park, MD 22030 USA

${ }^{7}$ NOAA National Environmental Satellite Data and Information Service, College Park, MD 20740 USA

${ }^{8}$ NASA Langley Research Center, Hampton, VA, 23681 USA

${ }^{9}$ NOAA National Weather Service National Centers for Environmental Prediction, College Park, MD 20740 USA

Corresponding authors: Daniel Tong (qtong@gmu.edu); Julian Wang (julian.wang@,noaa.gov) 

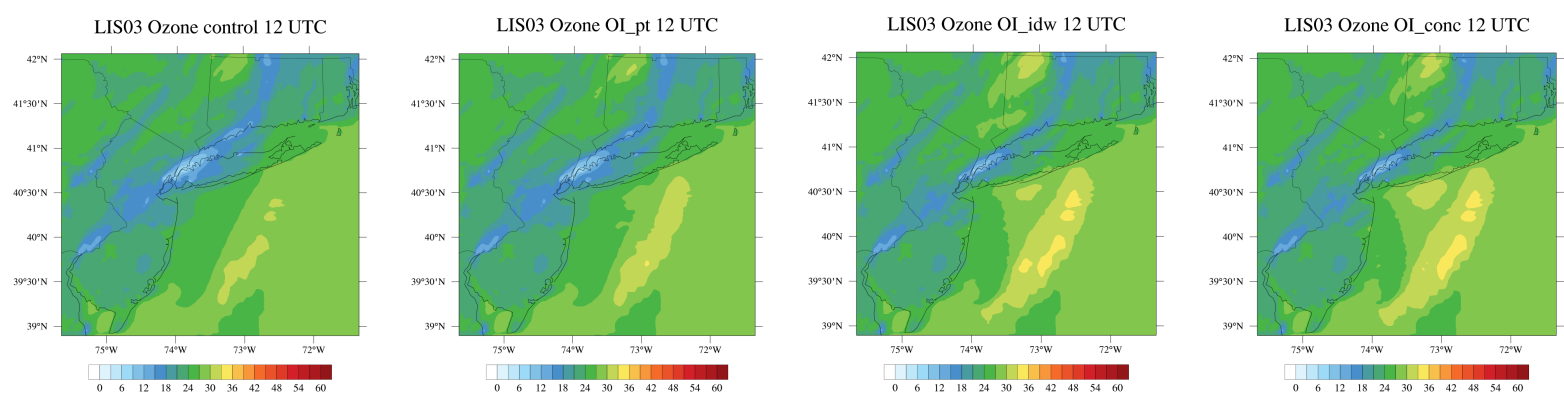

Figure S1: Spatial distributions of ozone simulated by Control, OI-pt, OI-idw and OI-conc at 0:00 and 12:00 UTC August 26, 2018.

Table S1: Regional mean statistical metrics between observed and simulated $\mathrm{O}_{3}$ and $\mathrm{NO}_{2}$ during the episode

\begin{tabular}{|c|c|c|c|c|c|c|c|c|c|c|c|}
\hline \multirow{2}{*}{$\begin{array}{l}\text { Ozone } \\
\text { CORR }\end{array}$} & \multirow{2}{*}{$\begin{array}{c}\text { Control } \\
0.81\end{array}$} & \multicolumn{2}{|c|}{$\mathrm{BCON}$} & \multicolumn{2}{|c|}{ NOAA } & \multirow{2}{*}{$\begin{array}{c}\mathrm{NO}_{2} \\
\mathrm{CORR}\end{array}$} & \multirow{2}{*}{$\begin{array}{c}\text { Control } \\
0.69\end{array}$} & \multicolumn{2}{|c|}{$\mathrm{BCON}$} & \multicolumn{2}{|c|}{ NOAA } \\
\hline & & 0.93 & $15 \%$ & 0.91 & $12 \%$ & & & 0.71 & $2 \%$ & 0.67 & $3 \%$ \\
\hline RMSE & 14.97 & 8.22 & $45 \%$ & 9.26 & $38 \%$ & RMSE & 4.12 & 3.82 & $7 \%$ & 4.98 & $21 \%$ \\
\hline NMB & -0.3 & 0.14 & $52 \%$ & 0.16 & $48 \%$ & NMB & -0.17 & -0.06 & $65 \%$ & -0.33 & $99 \%$ \\
\hline NME & 0.34 & 0.19 & $45 \%$ & 0.21 & $38 \%$ & NME & 0.35 & 0.33 & $4 \%$ & 0.43 & $23 \%$ \\
\hline
\end{tabular}

Table S2: Statistical metrics between airborne measured and simulated $\mathrm{NO}_{2}$ vertical column density on 28 and 29 , August 2018

\begin{tabular}{cccccccc}
\hline BOE & CORR & NMB & NME & NAQFC & CORR & NMB & NME \\
\hline 28 AM & 0.56 & 68.64 & 87.95 & 28AM & 0.44 & 47.83 & 89.48 \\
$28 \mathrm{PM}$ & 0.81 & 43.74 & 63.10 & $28 \mathrm{PM}$ & 0.79 & 76.43 & 80.08 \\
$29 \mathrm{AM}$ & 0.79 & 25.44 & 48.53 & $29 \mathrm{AM}$ & 0.41 & 11.91 & 69.95 \\
$29 \mathrm{PM}$ & 0.78 & 22.99 & 45.15 & $29 \mathrm{PM}$ & 0.65 & 43.99 & 64.60 \\
AVG & 0.74 & 40.20 & 61.18 & AVG & 0.57 & 45.04 & 76.03 \\
\hline
\end{tabular}

